\title{
The clinical cutting edge
}

\author{
As CRISPR-Cas9 enters human testing, the blistering pace of progress in preclinical research is offering a \\ bewildering array of options for therapeutic gene editing.
}

T his March, while the world was dealing with the onslaught of COVID19 , another kind of medical history was being made at the Oregon Health \& Science University Casey Eye Institute. Gene-editing pioneer Editas Medicine and Allergan announced that a patient had been dosed with the first ever CRISPRCas9-based in vivo therapy - a treatment for a form of Leber's congenital amaurosis. Just a few days earlier, positive results were published from the first clinical trial of T cells edited ex vivo using CRISPR-Cas9. At least 20 other human trials are underway to study the safety of CRISPR-Cas9 editing in humans, most of them relying on cellular DNA repair mechanisms to excise DNA in patient cells. With the rapid pace of research exploring an ever-growing array of engineered CRISPR enzymes and fusions, translational efforts will likely soon move toward a more ambitious goal: direct correction of genetic lesions.

The Focus in this issue explores the latest developments in CRISPR tools and therapies. Today, the ubiquity of these gene-editing reagents in laboratories around the world is a given. But it is remarkable that CRISPR-Cas9 therapies have entered human testing so soon after their initial discovery.

In 2012, the importance of CRISPR-Cas9 as a bacterial immune defense system for protecting against phage attack was just becoming clear. But no human cell had ever been exposed to CRISPR proteins by design. No one knew whether these prokaryotic proteins would work in human cells and what repair pathways would be engaged. No one knew whether the action of CRISPRCas9 would be toxic in human cells. And no one knew whether these large gene-editing proteins could be delivered to cells, tissues and organs inside the body.

In 2020, we have a better handle on these questions. We understand that CRISPRCas9 can create insertions/deletions (indels of 1-100 bp) or targeted insertions without homology ( $\geq 1 \mathrm{~kb})$ at one double-strand break (DSB) or targeted deletions between two DSBs (10 bp to several $\mathrm{kb}$ ) as a result of non-homologous end joining mediated by mismatch repair. In dividing cells, homology-directed repair (HDR) can also make targeted gene insertions via a DSB and donor construct. Preclinical studies are determining the effects of cell cycle phase, cellular development, differentiation, age or metabolic status on gene-editing activity. That said, CRISPR-Cas9's dependence on cellular repair enzymes - present at different levels at different times in different cells - and the difficulty of delivering components to the body mean gene editing in vivo remains a work in progress. (Intellia Therapeutics and Regeneron intend to test systemic injection using lipid nanoparticles for transthyretin amyloidosis later this year.)

In contrast, ex vivo gene editing is becoming routine. Using electroporation and ribonucleoproteins, CRISPR-Cas9 can generate indels via non-homologous end joining in human hematopoietic stem cells and primary $\mathrm{T}$ cells with efficiencies exceeding $80 \%$. Even the holy grail of gene correction via HDR can reach frequencies higher than $30 \%$ in dividing cells.

There is also growing optimism that a burgeoning set of Cas proteins (such as SaCas9, Cas12a, Cas12b, SpRY and Cas9- $-\mathrm{Sc}^{++}$) and their engineered variants partnered with enzyme fusions can take gene editing to new heights, addressing off-target effects, PAM restrictions, activity windows, gene correction inefficiencies and the lack of HDR in non-dividing cells (for example, postmitotic neurons).

DNA base editing - a process first described in 2016 that uses catalytically inactive Cas9 or Cas9 nickase fused to a deaminase (and, in the case of cytidine base editors, an additional DNA glycosylase inhibitor fusion) - allows the direct conversion of a single nucleotide into another without a DSB. So far, options are limited to C-to-T and A-to-G changes, but other base editors are under development. Single point mutations contribute about half of all known pathogenic mutations, and current base editors can address $\sim 60 \%$ of disease-related single nucleotide polymorphisms ( $\sim 50 \%$ of all mutations in ClinVar). Clinical programs for the translation of base editors into therapeutics are underway at Beam Therapeutics, which plans to file its first investigational new drug application next year.

If more than single nucleotide changes are needed, one recent solution may come in the form of prime editing. This approach exploits reverse transcriptase fused to a Cas9 nickase to replace part of the sequence around a single-strand cut using an extended guide RNA. So far, the method has been shown to install all possible single-base-pair mutations and short indels ( $<100 \mathrm{bp})$. It promises greater targeting flexibility and editing precision than base editing, albeit with a lower editing efficiency so far and increased indel byproducts. Clinical development is underway at the startup Prime Medicine.

Two other groups of enzymes that offer the promise of more predictable gene editing are transposases (enzymes that catalyze the insertion of mobile DNA elements into host DNA) and recombinases (enzymes that catalyze the rearrangements of DNA segments). Thus far, engineering sequence specificity without compromising activity has proven elusive for recombinases, although transposases associated with Cas12k or the CRISPR-Cascade system have now been engineered to target arbitrary sequences and catalyze insertion of up to several kilobases of DNA in bacteria.

A completely different approach - which avoids concerns about off-target changes permanently made to the DNA blueprint - is to use the CRISPR machinery to alter RNA, which degrades over time. In addition to several approaches to co-opt endogenous ADAR (adenosine deaminase acting on RNA), dCas13b can be fused to ADAR directly to induce adenosine-to-inosine editing at desired sites. Several companies, including Locana, Beam, Korro Bio, ShapeTx, EdiGene and ProQR, are pursuing RNA-editing approaches.

All in all, the sheer abundance of targeted approaches coming online to manipulate nucleic acids in cells is astonishing. Together with zinc finger endonucleases, transcription activatorlike and effector nucleases (TALENs) and meganucleases, CRISPR-Cas promises patients an increasing array of gene-editing options to treat previously intractable and highly penetrant genetic disease.

Published online: 8 July 2020

https://doi.org/10.1038/s41587-020-0612-2 